\title{
Web et musées : le choc des cultures
}

\section{Samuel Bausson et Francis Duranthon}

URL : http://journals.openedition.org/ocim/1291

DOI : 10.4000/ocim.1291

ISSN : 2108-646X

\section{Éditeur}

OCIM

Édition imprimée

Date de publication : 29 novembre 2013

ISSN : 0994-1908

\section{Référence électronique}

Samuel Bausson et Francis Duranthon, «Web et musées : le choc des cultures », La Lettre de l'OCIM [En ligne], 150 | 2013, mis en ligne le 29 novembre 2015, consulté le 30 avril 2019. URL : http:// journals.openedition.org/ocim/1291; DOI : 10.4000/ocim.1291

Ce document a été généré automatiquement le 30 avril 2019

Tous droits réservés 


\title{
Web et musées : le choc des cultures
}

\author{
Samuel Bausson et Francis Duranthon
}

1 Traditionnellement, un musée pratique une communication descendante, des " sachants » vers les « profanes ». Autour de ses collections qui lui donnent sa légitimité, il construit un discours expert qu'il délivre sous la forme qu'il juge la mieux adaptée à ses publics. Le développement et l'évolution du numérique nous obligent à reconsidérer un certain nombre de paradigmes, tant dans notre relation au public que dans nos modes d'organisation du travail.

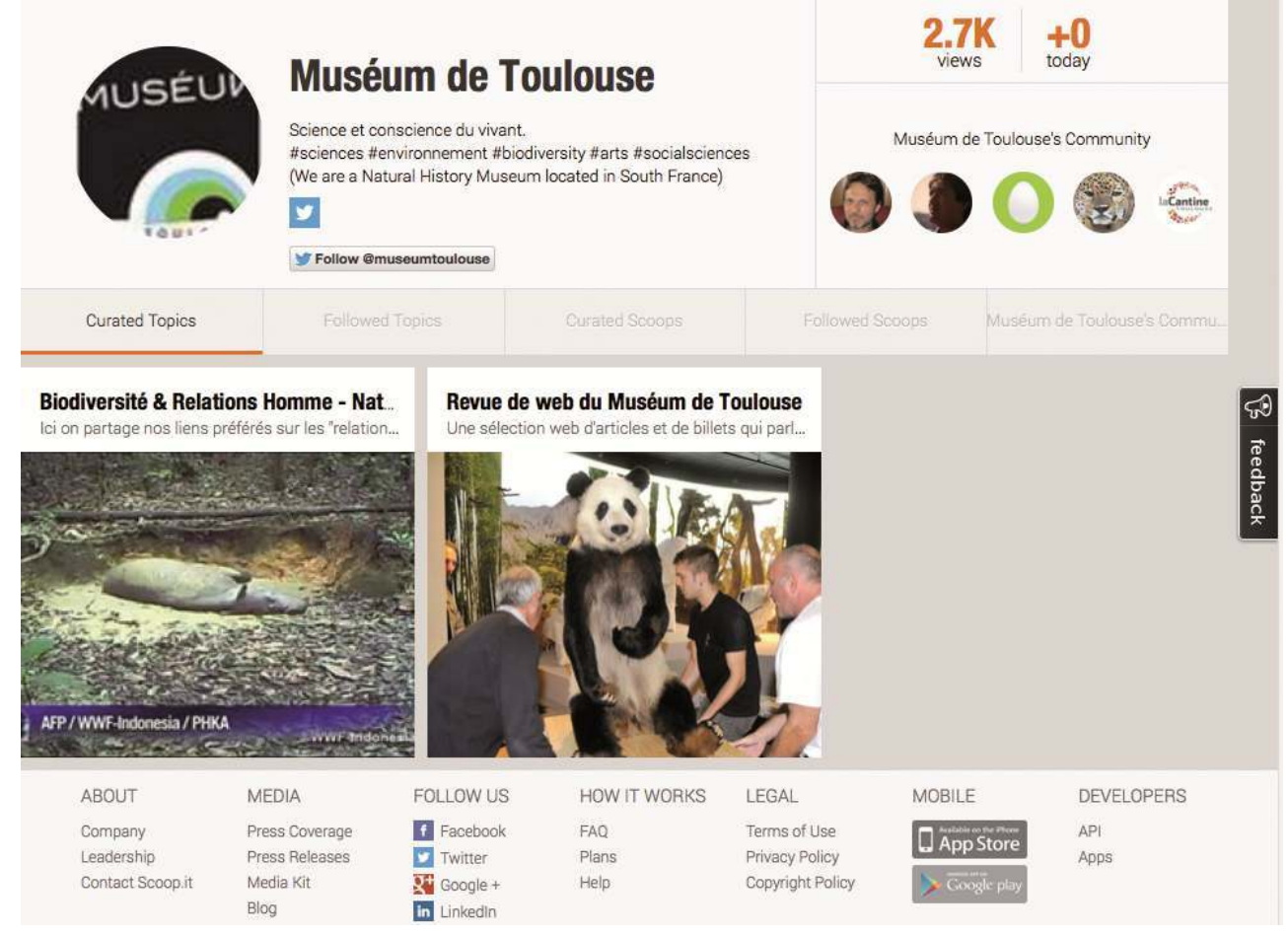

La communauté du muséum de Toulouse sur Internet

(C) MUSÉUM DE TOULOUSE 
2 Pour développer cette contribution, nous adopterons le principe d'une comparaison, un vis-à-vis, entre le modèle de fonctionnement des musées et celui des communautés Web ouvertes et contributives Il s'agit d'une comparaison schématique et délibérément «simplificatrice» pour les besoin de l'exercice. L'idée est avant tout d'avoir une idée générale de ce qui différencie les deux modèles et surtout d'en saisir les enjeux et les leviers de changements pour les institutions.

\section{Logiques générales}

3 Jusqu'à présent, la majorité des établissements est entrée dans une logique "vitrine » diffusionniste faite pour rayonner, pour asseoir l'autorité de l'établissement et le placer au centre d'une position de prestige. Ceci s'oppose aux pratiques des communautés contributives basées sur des logiques de co-production ouverte et « relationnelle » (de contributeurs en réseaux) de savoirs-communs (« commons »).

\section{Valeur de la rareté ou valeur de l'abondance}

Dans nos modes de fonctionnement habituels, la production de contenus est basée sur une forme de rareté qui en fait la valeur. Ces contenus se développent à travers nos expositions, qu'elles soient temporaires ou permanentes, mais aussi sur les pages de nos sites Web ou au travers des différentes offres d'animation et de médiation. Elle repose sur l'expertise de «sachants" (conservateurs, comités scientifiques...) grâce auxquels l'établissement porte sa parole vers son public, définissant des cibles qu'il veut atteindre et adaptant son discours en conséquence.

5 Dans la logique des réseaux sociaux, c'est l'échange qui produit la valeur. Par le développement d'une architecture participative de type « 2.0 », cette valeur se caractérise par la production de «biens communs» (qui correspondent au «bien » défini par la communauté productrice). Dans ce schéma, on se place alors dans une économie de l'abondance, abondance d'idées et de production numériques où la reproduction n'engendre pas de coût supplémentaire.

\section{Logique vitrine (inside-out) versus logique contributive en réseaux (outside-in)}

6 Ces dernières années, une logique de marque, poussée en avant par les modèles économiques dominants, s'est fortement développée au sein des grandes institutions culturelles : Le Louvre-Lens, Le Louvre-Abu Dhabi, Pompidou-Metz, Guggenheim-Bilbao... Cette marque se construit à partir d'un centre rayonnant, l'établissement d'origine, qui affirme son identité et gagne de la visibilité (des parts de marché) à travers un portail ou une porte d'entrée unique (la Marque) pour faire venir, attirer, rayonner et développer son prestige. Le public y est traité comme un visiteur éphémère que l'on veut fidéliser pour en faire un client, un consommateur des productions de l'établissement que l'on va qualifier pieusement d'audience ou d'usagers. Nous sommes ici face à une logique vitrine (inside-out) où le message part de l'établissement vers ses visiteurs.

7 Dans la logique relationnelle (outside-in) promue par les réseaux sociaux, l'identité est co-construite pour et par les membres d'une communauté. Les liens se font par affiliation, 
par affinité d'intérêts ou de pratiques. On se « reconnaît» par "profils" autour de centres d'intérêts communs et partagés. On développe ainsi une économie « relationnelle » où l'intelligence du groupe ou entre les profils (ex : recommandation sociale) produit plus de valeur que chaque individu séparément ne le fait par lui-même. On ne parle plus de visiteur mais de membre d'une communauté, à la fois utilisateur et contributeur d'un « bien commun », d'un « savoir commun ».
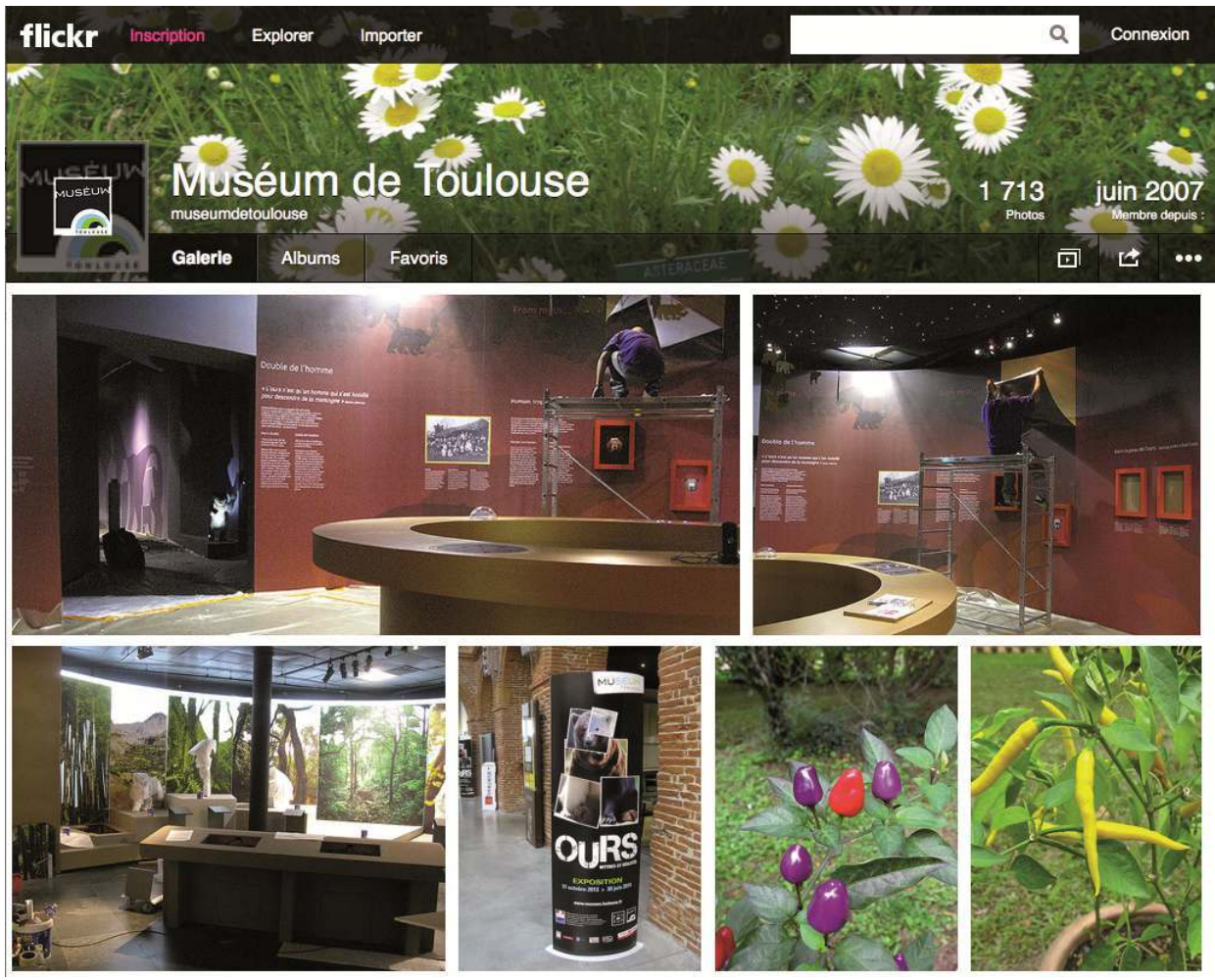

Le compte Flickr du muséum de Toulouse rassemble des photographies envoyées par les internautes. (C) MUSÉUM DE TOULOUSE

\section{Principes généraux}

8 Selon les modèles dominants, les contenus des productions des établissements culturels sont généralement protégés par des droits de propriété intellectuelle rigides qui donnent au producteur une exclusivité d'usage sur les contenus qu'il produit. Nous sommes dans un système fermé, dans une économie qui limite la reproduction des contenus et en assure la diffusion de manière contingentée.

9 Par opposition, dans le système ouvert que prônent les communautés contributives, les contenus sont ouverts (licences ouvertes, licences libres). À l'instar des logiciels libres où le code source est accessible et modifiable, on peut refaire, on peut apprendre, on peut améliorer selon ses besoins et les contenus peuvent être copiés, modifiés et redistribués (mixages, mashups...). La notion d'ouverture est le «ressort» central du modèle contributif. Elle autorise la diffusion et la reproduction des contenus en abondance. 


\section{Exclusivité versus inclusivité (individus)}

10 À l'exclusivité (cohérente avec la logique inside-out), aux droits d'entrées (clubs), à la sélection sur le statut, aux candidatures a priori s'oppose l'inclusivité par principe des communautés ouvertes. Toute personne partante pour contribuer n'a pas de statut particulier à justifier au préalable. Les communautés d'échanges et de travail collaboratif sont basées sur la rencontre d'individus volontaires (outside-in). Les plateformes en ligne disposent d'architectures participatives et des mécanismes pour faciliter l'entrée dans la communauté, minimiser l'effort pour y participer, et autoriser une contribution maximale, dans une logique d'abondance.

\section{"Lecture » (à sens unique) versus « Lecture-Écriture » (ReadWrite Web - Participatif/ Contributif)}

11 En contrepoint du modèle diffusionniste (cohérent avec la volonté de rayonnement) à sens unique où l'audience est consommatrice («lectrice») de contenus, la capacité "d'écriture » (Read-Write), du copier-coller, de la rediffusion, du mixage, de l'annotation, de la co-édition, de la co-création caractérise les communautés contributives. De la simple « conversation » à la co-création (où tous les acteurs négocient ouvertement les règles de fonctionnement et le projet contributif au départ) l'échelle de la participation est large. Ce principe moteur est au cœur de «l'économie » ouverte et contributive.

\section{Modèle structurel et modes opérationnels}

Deux modèles organisationnels s'opposent: la cathédrale versus le "bazaar " pour reprendre la métaphore de la logique de production du logiciel libre de Éric S. Raymond, ou, formulé autrement, la pyramide versus le "réseau de pair à pair ». Ces deux modèles peuvent se combiner en alternance, sans s'exclure.

\section{La cathédrale}

La combinaison des principes "top down" (à sens unique) et d'une centralisation (fermée) donne une hiérarchie sur le modèle pyramidal. La gouvernance globale est exercée par une autorité de « statut » basée sur une expertise établie au préalable. Du fait de la structure hiérarchique, il en résulte un cloisonnement métier, avec une spécialisation et une valorisation de l'expertise. Dans ce type de structure, chacun se focalise sur son expertise en répondant à son supérieur hiérarchique, l'expert "métier " se focalisant sur une partie technique et spécifique d'un projet. 


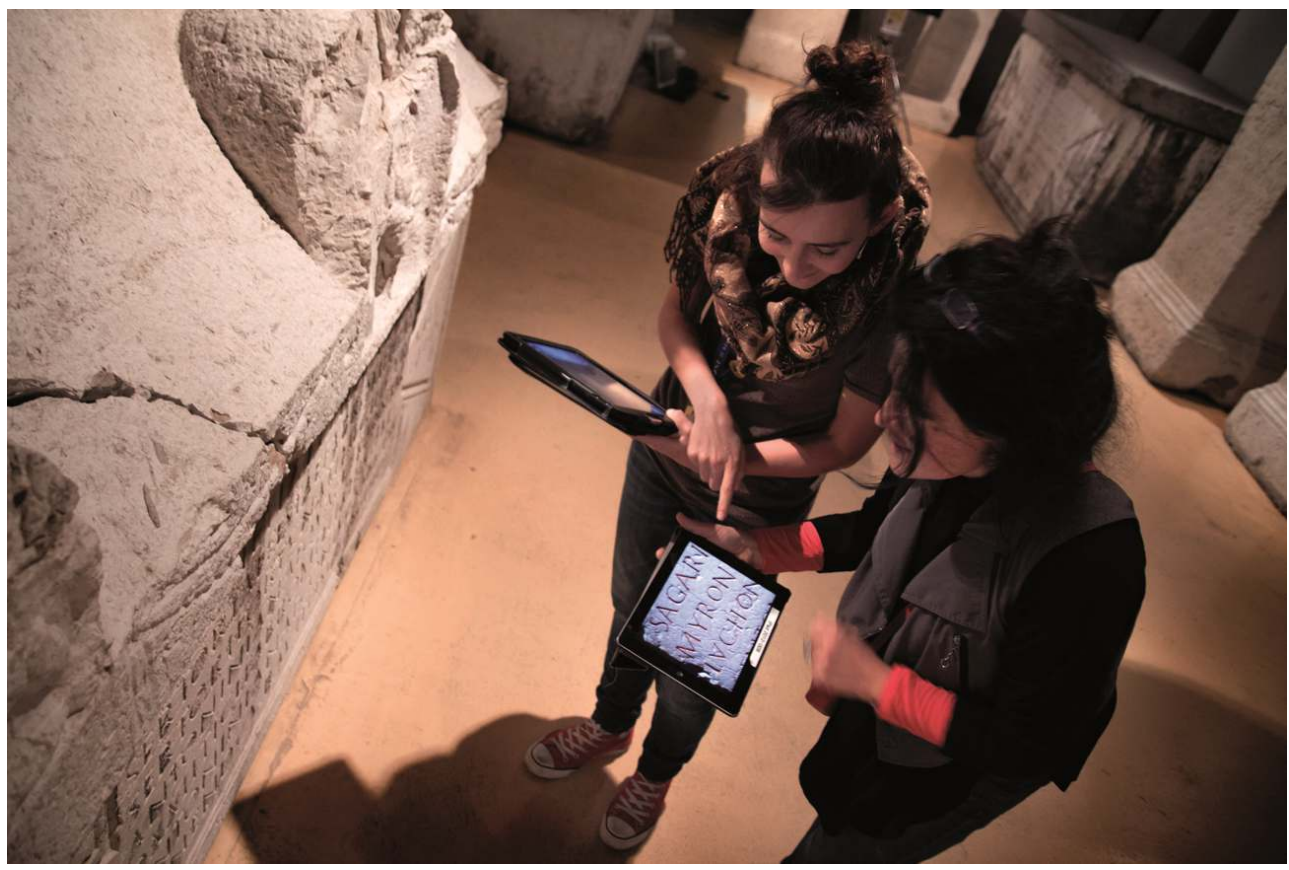

Pour l'événement Museomix qui s'est déroulé à Lyon au musée gallo-romain de Fourvière en 2012, le projet fil rouge des Wifius est une expérience collaborative.

(c) MUSEOMIX

\section{Le « bazaar »}

14 Ce système d'organisation de pair à pair, est lui, par essence, décloisonné. La décentralisation et l'horizontalité donnent un réseau d'interconnexions de pairs à pairs. La gouvernance globale est basée sur la contribution des pairs, amateurs (celui qui aime) ou experts professionnels. L'autorité et la visibilité au sein de la communauté s'acquièrent a posteriori par la contribution apportée et par l'historique de ses actions («actioncratie »). Les conflits y sont réglés en priorité par consensus.

\section{Processus de fonctionnement}

\section{Organisation statique versus écosystème émergent}

Une structure pré-établie, comme celle qui prévaut dans nos musées, est bâtie pour être la plus stable possible. Elle vise sa pérennité en tant que telle et s'oppose à une structure organique qui évolue, à un écosystème émergent qui s'adapte de façon modulaire en fonction des projets, des besoins, des acteurs. Par essence, dans ce type d'écosystème auto-organisé, le focus se fait sur le projet à développer plutôt que sur la structure.

\section{Linéarité versus itérations « Enlightened trial and errors is better than long planning » (Ideo)}

Nos modes traditionnels de développement de projet sont linéaires, empruntés au monde de la construction. C'est un modèle séquentiel de conception-production (comité de direction $>$ comité projet $>$ comité technique $>$ cahier des charges $>$ développement- 
production > livraison). On y passe par des phases précises (APS, APD...) sans retour en arrière.

17 À l'inverse, le modèle collaboratif fonctionne avec des allers-retours selon le principe du "livrer tôt, livrer souvent ", où des versions minimales viables sont mises à l'épreuve des publics dès que possible, où les erreurs (mesurées) sont considérées comme des étapes d'a justements naturelles et une condition pour la «réussite » finale. Un processus où le «faire » (la confrontation avec la réalité du projet en contexte, avec les opportunités et les limites des outils utilisés) est porteur de nouvelles idées de conception, de remise en question des présupposés initiaux. C'est le modèle de l'alpha-béta dans le logiciel et du versionning historique dans les wiki-documents ${ }^{1}$.

\section{Valider pour faire versus faire pour valider}

Dans notre mode de fonctionnement actuel, le management dit ce qu'il faut faire (cohérent avec le positionnement de contrôle) et l'expert qui a l'autorité est le «gardebarrière ». Il « valide » les initiatives.

À l'inverse, dans le modèle collaboratif, «celui qui fait a raison " (jusqu'à preuve du contraire), celui/celle qui veut quelque chose l'entreprend (le fait ou aide d'autres à le faire avec lui/elle). La validation est posée par la confiance a priori de la part des membres de la communauté et acquise par l'historique des actions précédentes (importance de la légitimité). La contribution à la communauté donne la légitimité d'en treprendre, de modifier, d'évaluer (et de critiquer).

Dans les deux cas (production de contenus et actions) la « charge » de la validation et le souci de la correction ne reposent plus sur celui/celle qui produit et est dans l'action, mais sont reportés sur celui/celle qui n'est pas en accord avec ce qui est produit. Il s'agit de libérer l'action, de faciliter la prise d'initiative et de créer des résultats en abondance. Le pari, c'est qu'au final il y aura plus de choses intéressantes produites qu'en faisant " attention » à vérifier qu'elles le soient avant de les entreprendre (en cohérence avec le positionnement d'ouverture initial). 


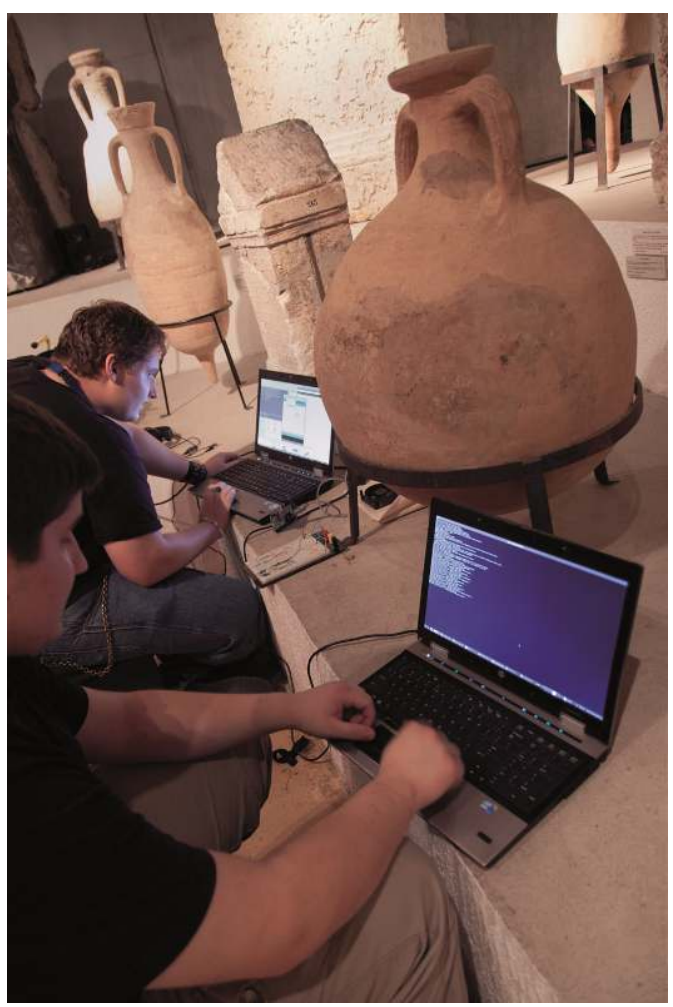

Le commerce méditerranéen antique nécessite quelques Arduino (circuit imprimé en matériel libre utilisé pour construire des objets interactifs indépendants) pour être mis en valeur.

(c) MUSEOMIX

\section{Changer les musées en bien communs?}

21 Les principes de fonctionnements des bibliothèques, des musées, des archives... ont été élaborés dans un cadre culturel et technique qui a évolué depuis. Les musées et les bibliothèques veulent de plus en plus ouvrir leur structure, encourager les visiteurs à y prendre une place plus importante, ouvrir leurs contenus et inciter les utilisateurs à se les rapproprier. Si nous sommes plus ou moins d'accord sur ces objectifs, sur le « quoi », c'est plutôt le «comment» qui nous échappe. Nous éprouvons une difficulté à impulser les changements nécessaires pour y parvenir concrètement. Nous initions pourtant des opérations dites «participatives ». Mais elles sont bien souvent ni tout à fait ouvertes, ni tout à fait engageantes auprès de visiteurs sur lesquels le musée a tendance à vouloir "garder la main». Ces opérations restent souvent sans impact à long terme dans l'intégration à part entière des visiteurs comme acteurs de la vie du musée, dans son déroulement, dans ses projets.

Cette notion d'écosystème des réseaux est intéressante quand on la met en vis-à-vis avec le fonctionnement de nos structures où le rôle de chacun est préétabli, cloisonné, et fixé dans un organigramme hiérar-

chique. Les musées n'ont pas cette dimension «organique » dans leurs ADN, d'où leur difficulté à s'adapter, à évoluer, à innover vers des solutions "agiles", ouvertes, cocréatives, plus adaptées aux usages de «nos usagés ».

Le défi qui nous attend dans les prochaines années est immense. Il s'agit ni plus ni moins d'un changement de paradigme. 
25 Et si on imaginait nos lieux de savoir comme des plateformes ouvertes d'une culture coproduite et partagée de pair à pair ? Et si on transformait radicalement nos structures en plateformes, supports d'une culture ouverte, distribuée et basée sur la co-construction d'un bien (culturel) commun ? Et si on imaginait des musées « ouverts » (aux espaces, aux contenus ouverts, où l'on peut se brancher comme sur une $\mathrm{API}^{2}$ ), des musées Read-Write (de co-création, de contributions), des musées P2P (en réseaux décentralisés) ${ }^{3}$ ?

Pour cela, les structures culturelles pourront s'inspirer des modes de fonctionnement en réseaux ouverts et contributifs. Il s'agira alors de sortir des logiques classiques de "diffusion", de passer d'une logique «vitrine » à une logique "relationnelle " (en réseaux). Il ne s'agira plus d'imaginer des musées "participatifs" mais, en allant beaucoup plus loin, d'imaginer des musées co-créatifs.

27 Cela impliquera de déplacer l'autorité du contrôle vers l'ouverture, de passer du musée rayonnant au musée "centré visiteur", de la validation vers la facilitation, de la captation vers la mise en réseau. Cela impliquera de modifier les critères d'évaluation, souvent restreints à de la fréquentation, pour des indices d'impact sur le « bien (culturel) commun ».

\section{Comment ?}

En la matière, tout est à inventer. Samuel Bausson, community manager du muséum de Toulouse, y réfléchit depuis longtemps et cherche à le mettre en pratique avec toute une communauté de contributeurs notamment avec le projet Muséomix. De ces expériences, ils tirent quelques pistes innovantes à suivre pour des établissements qui souhaiteraient s'engager sur cette voie de musées co-créatifs.

\section{Des salons-labos dans les musées}

Comment faire pour changer les musées dans ce sens ? Cela pourrait se faire en intégrant des « zones d'autonomie » dans les musées et les bibliothèques :

- des laboratoires d'expériences (en ligne et sur place) ouverts et modulaires où les communautés créatives peuvent venir développer leurs propres projets, en affinité avec les thématiques du lieu;

- des points de rendez-vous où les amateurs ou professionnels (dont les acteurs du musée font partie) peuvent faire connaissance avec des pairs partageant les mêmes intérêts qu'eux ;

- des «salons » où ils peuvent se retrouver, échanger sur leurs idées et leurs pratiques et faire émerger de nouveaux projets

- des « ateliers de bricolage » où ils peuvent les prototyper et les fabriquer ensemble.

Autrement dit, il s'agit de changer les musées et les bibliothèques en plateformes qui génèrent de l'affinité, de l'affiliation, et de la créativité.

31 Mais ce changement sera sans doute plus fortement impulsé en marge des institutions. Museomix est un exemple qui va dans ce sens. C'est une expérience pour tester des modalités d'ouverture du musée aux communautés créatives. Une expérience qui démontre que ces communautés sont désireuses d'entrer dans le musée, d'y prendre une place à part entière si le musée sait les laisser libre. Par son format événementiel, Museomix ne dure que quelques jours. C'est une façon de convaincre l'institution de lâcher prise, sur une durée limitée «réconfortante». Mais une fois la preuve par 
l'exemple faite, l'ambition est d'inciter et d'inspirer de nouvelles formes qui s'installent dans la durée.

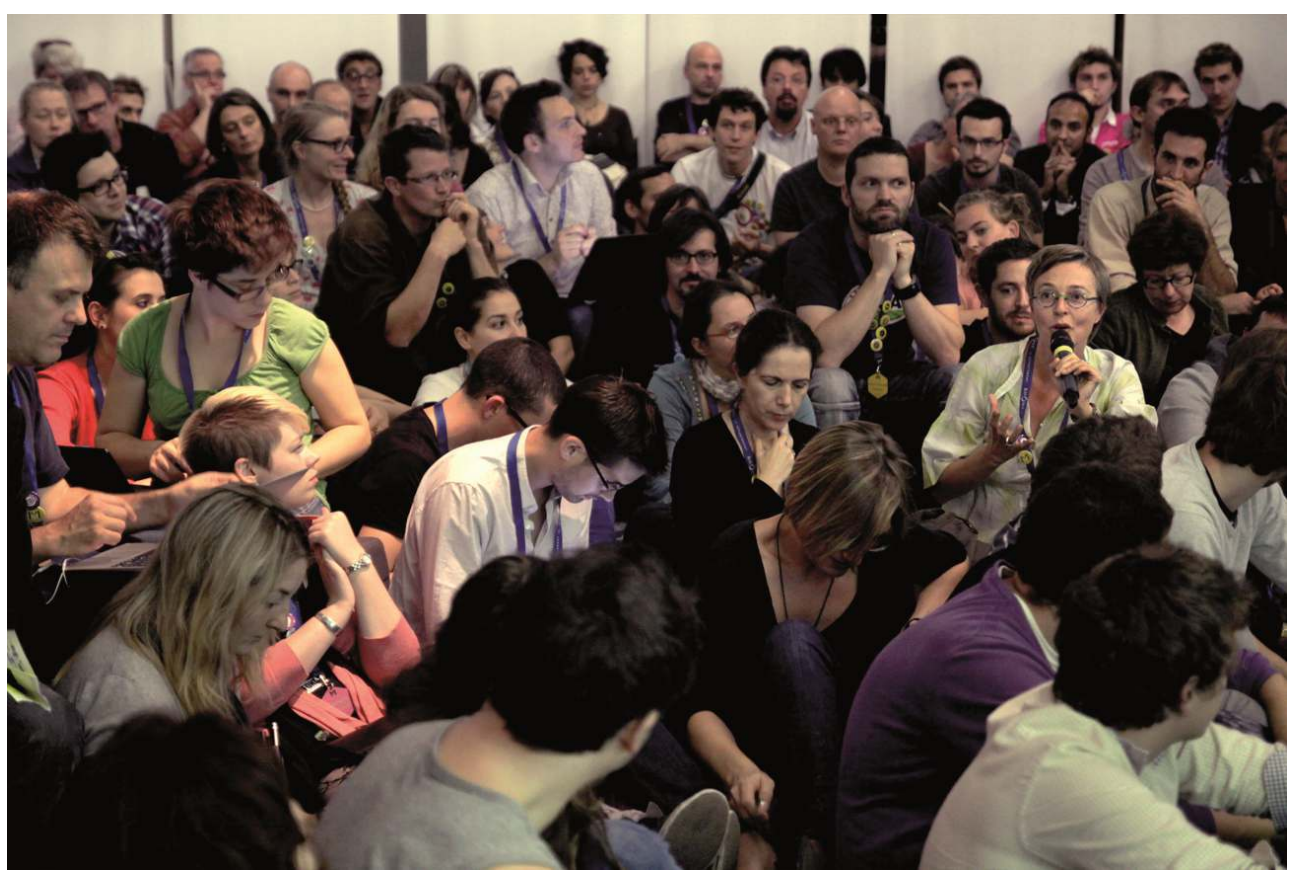

Échanges des participants à Museomix lors d'une réunion plénière

(C) MUSEOMIX

\section{Changer pour des musées et des bibliothèques essentiels à nos vies}

Ni club d'experts, ni cafés du commerce, les musées et les bibliothèques peuvent devenir des carrefours de rencontre avec des personnes hors de nos cercles habituels, des agoras pour échanger des idées nouvelles, bousculer nos certitudes, stimuler notre imagination. Ils offrent un cadre privilégié pour échanger en intelligence et de manière apaisée, instruite, curieuse, et créative. Ils peuvent devenir des terrains d'expérimentation pour partager des expériences de co-création inédites et ouvrir de nouvelles possibilités d'invention de ce qui fait une culture partagée, une culture commune.

Les communautés contributives nous montrent une façon d'y arriver de façon plus agile.

\section{BIBLIOGRAPHIE}

Sites Internet

- http://internetactu.blog.lemonde.fr/2013/04/05/du-biais-de-linternet-centrisme-au-risque-dusolutionnisme/

- http://en.wikipedia.org/wiki/Michel_Bauwens 
- Le peer-to-peer, clef de voûte pour les économies futures ? http://adam.hypotheses.org/1143

- Bauwens à la Cantine : http://lacantine.ubicast.eu/videos/webinar-16-12-2011-141701/

- http://en.wikipedia.org/wiki/Henry_Jenkins

- http://fr.wikipedia.org/wiki/Bernard_Stiegler

- http://owni.fr/2011/11/30/vers-une-economie-de-la-contribution/

- http://storify.com/Bpi/pour-en-savoir-plus-sur-les-biens-communs

\section{NOTES}

1. Processus permettant de conserver une trace des modifications successives apportées à un fichier numérique (documentation, code source, base de données) d'un site Internet dont les pages sont modifiables par le visiteur.

2. API : interface de programmation clairement délimitée par laquelle un logiciel offre des services à d'autres logiciels.

3. P2P (peer-to-peer ou pair-à-pair) système qui permet à plusieurs ordinateurs de communiquer via un réseau, de partager des fichiers, de flux multimédia.

\section{RÉSUMÉS}

Face au développement des nouvelles pratiques des internautes (réseaux sociaux), les auteurs proposent quelques pistes innovantes à suivre par les institutions muséales qui souhaiteraient passer d'une logique « vitrine » à une logique « relationnelle » et imaginer des musées co-créatifs dans lesquels le visiteur - intégré comme acteur de la vie du musée et de ses projets - devient membre d'une communauté, à la fois utilisateur et contributeur d'un savoir commun.

\section{INDEX}

Mots-clés : Musées, web

\section{AUTEUR}

\section{SAMUEL BAUSSON ET FRANCIS DURANTHON}

Samuel Bausson est community manager et webmaster du muséum d'Histoire naturelle de Toulouse. samuel.bausson@mairie-toulouse.fr Francis Duranthon est conservateur en chef, directeur du muséum d'Histoire naturelle de Toulouse. francis.duranthon@mairie-toulouse.fr 